\title{
Preface for the Special Issue of Green Smart Manufacturing
}

\author{
József Váncza ${ }^{1} \cdot$ Sang Do Noh ${ }^{2} \cdot$ Hae-Sung Yoon ${ }^{3}$
}

Published online: 1 May 2020

(c) Korean Society for Precision Engineering 2020

In the last few decades, significant efforts have been directed towards the Green and Smart Manufacturing. Societies have urged manufacturing industries to take greater responsibilities in lowering environmental burdens. Thus, Green Manufacturing researchers have investigated the solution to reduce energy consumption, improve manufacturing efficiency, and decrease environmental wastes in processes, machines, and factories. Sometimes the overall process chain is analyzed at a higher level, and interactions between process chains are controlled as well as the optimization of a single manufacturing process. One of the key abilities for Green Manufacturing is to assess and predict the environmental influences of individual components or processes composing the manufacturing system.

At the same time, Smart Manufacturing has been spotlighted to optimize manufacturing systems with enhanced flexibility. Smart Manufacturing, broadly speaking together with the 4th Industrial Revolution, refers to fully integrated, collaborative manufacturing systems mainly for real-time response to rapidly changing markets. By connecting manufacturing processes, machines, factories, and people, Smart Manufacturing shows great potentials in improving overall manufacturing performance and enhancing the flexibility of the system. Furthermore, it is highly relevant to Green

József Váncza

vancza@sztaki.mta.hu

Sang Do Noh

sdnoh@skku.ac.kr

$\triangle$ Hae-Sung Yoon

hsyoon7@kau.ac.kr

1 Institute for Computer Science and Control, Hungarian Academy of Sciences, Kende u. 13-17, Budapest XI 1111, Hungary

2 Department of Systems Management Engineering, Sungkyunkwan University, 2066 Seobu-ro, Jangan-gu, Gyeonggi-do 16419, Republic of Korea

3 School of Aerospace and Mechanical Engineering, Korea Aerospace University, 76 Hanggongdaehak-ro, Deogyang-gu, Goyang-si, Gyeonggi-do 10540, Republic of Korea
Manufacturing, as it can manage the environmental damage not only during the manufacturing process but also over the whole product lifecycle. The data obtained from the process and the decision-making system are widely utilized for sustainability by minimizing energy consumption/wastes and improving the efficiency of manufacturing activities.

However, unlike the Industrial Revolution in the past, Smart Manufacturing is enabled by the implementation and integration of various technologies. To collect and utilize the data in manufacturing, huge numbers of sensors are usually embedded with network communication systems. Collaborative robots are commonly adopted in automated processes. For real-time response, processing and analysis of big data are essential, and artificial intelligence (AI) is actively investigated in many leading companies. Based on processed data, cyber physical system (CPS) reflects the physical world to manage the current manufacturing system, and further to forecast the manufacturing performances in the dynamic market. For effective and efficient Smart Manufacturing, it is necessary to encourage synergy between these technologies through the appropriate utilization.

Recently, Green Smart Manufacturing is strongly driven by governments, and there has been a significant increase both in research activities and industrial applications. Policies such as German Industry 4.0, Remaking America and Made in China 2025 provide supporting evidence that many manufacturing countries have a strong willingness toward Green Smart Manufacturing. However, though a lot of research and case studies, it is still not straightforward to plan and design Green Smart Manufacturing in a single specific form. Configurations and characteristics of Green Smart Manufacturing may vary referring to different industries, and performances of the manufacturing system need to be carefully assessed concerning overall efforts and time for installing and operating the system.

Thus, it is required to overview novel technologies and practical case studies to circulate and further develop research ideas. This special issue will provide a timely appropriate snapshot of the latest research and case studies 
on Green Smart Manufacturing. Specifically, the following topics will be covered:

- Artificial intelligence (AI) and big data analysis in green manufacturing

- Digital twin, cyber physical system (CPS), mixed reality (MR) applications

- Appropriate sensors, monitoring systems, and prognostics and health management (PHM)

- Network communication systems, network security and $5 \mathrm{G}$ applications

- Green applications of additive manufacturing, collaborative robotics and soft robotics

- Case studies of smart factory for energy saving or reduction in environmental impact

We would like to express our sincere appreciation to all the authors as well as editorial members for their precious time and effort, and to Ms. Ji Young Oh of the Korean Society for Precision Engineering (KSPE) for her dedicated support during the review and production processes. We are also grateful to Prof. Sung-Hoon Ahn (Seoul National University, Korea), Editor-in-chief of IJPEM-GT, for giving us the opportunity to publish this special issue.

Publisher's Note Springer Nature remains neutral with regard to jurisdictional claims in published maps and institutional affiliations.

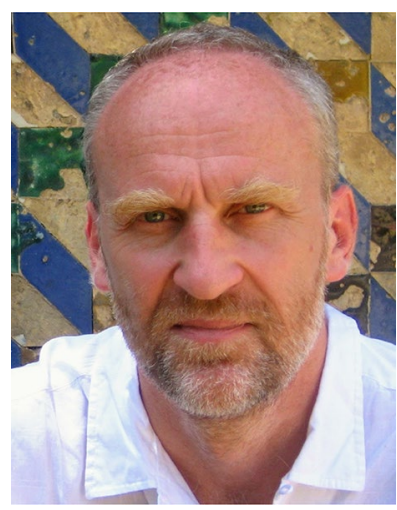

József Váncza is the Head of the Research Laboratory on Engineering and Management Intelligence (EMI) at MTA SZTAKI, The Institute for Computer Science and Control of the Hungarian Academy of Sciences, Budapest, Hungary. He received an M.Sc. degree in Electrical Engineering (1984) and a Ph.D. degree in Mechanical Engineering (1994). He has been lecturing for three decades at the Budapest University of Technology and Economics, Hungary, where he is an Associate Professor at the Department of Manufacturing Science and Technology. For his research and educational activities József Váncza was awarded the Knight Cross of the Order of Merit of the Hungarian Republic. He has published 190 scientific papers that received $2000+$ independent citations. His main research interests include Computational Intelligence, Advanced Planning in Production and Energy Management, and has special interest in Cooperative and Sustainable Production.

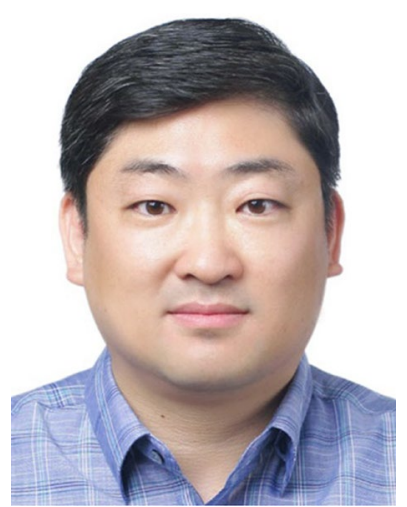

Sang Do Noh is currently a Full Professor in the Department of Systems Management Engineering at Sungkyunkwan University. He received his undergraduate degree in Mechanical Engineering from Korea Advanced Institute of Science and Technology (1992) and his Masters' degree and Ph.D. in Manufacturing Engineering from Seoul National University (1999). Since then, he has held professional and visiting positions at Institute for Advanced Engineering, GM Global R\&D Center, and Royal Institute of Technology/Mälardalen University. He joined Sungkyunkwan University in 2002. Dr. Noh's research interests include CAD/ CAPP/CAM, Digital Virtual Manufacturing, PLM, Smart Manufacturing, Smart Factory, CPPS, and Digital Twin.

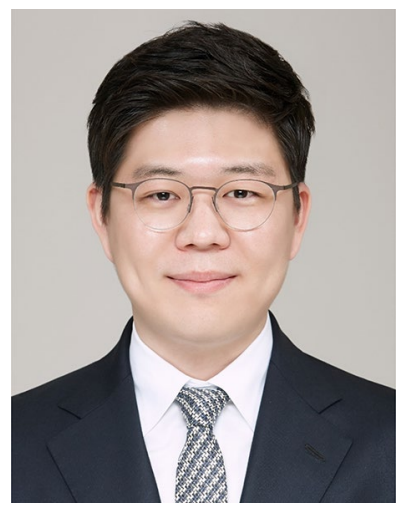

Hae-Sung Yoon is currently an Assistant Professor in the School of Aerospace and Mechanical Engineering at Korea Aerospace University (KAU). He received his B.S. in 2010, and Ph.D. in 2015 (M.S. integrated) all in Mechanical and Aerospace Engineering from Seoul National University, Korea. Since then, he has held professional positions as a Postdoctoral Fellow in BK21+ Program at Seoul National University from 2015 to 2016, and as a Research Associate in the Department of Mechanical Engineering at University of Wisconsin-Madison, USA, from 2016 to 2018. Yoon's research interests are in Hybrid/Smart Manufacturing, Sustainable Manufacturing, Ultra precision Machining, Micro/Nano Fabrication, 3D/4D Printing, Manufacturing for Design, and Smart Materials. 